\title{
A high-fructose diet induces epithelial barrier dysfunction and exacerbates the severity of dextran sulfate sodium-induced colitis
}

\author{
KATSUTO KAWABATA, SHUJI KANMURA, YUKO MORINAGA, \\ AKIHITO TANAKA, TOMOAKI MAKINO, TOSHIHIRO FUJITA, SHIHO ARIMA, \\ FUMISATO SASAKI, YUICHIROU NASU, SHIROH TANOUE, SHINICHI HASHIMOTO and AKIO IDO \\ Digestive and Lifestyle Diseases, Kagoshima University Graduate School of Medical \\ and Dental Sciences, Kagoshima 890-8544, Japan
}

Received August 13, 2018; Accepted November 19, 2018

DOI: $10.3892 / \mathrm{ijmm} .2018 .4040$

\begin{abstract}
Excessive fructose intake is a risk factor for gut symptoms in patients with inflammatory bowel disease, however, its effect on the intestinal tract has not been evaluated previously. The present study investigated the impact of a high-fructose diet (HFD) on intestinal barrier function in mice with experimental colitis. C57/BL6 mice were provided with either a HFD or control diet and either plain drinking water or water containing $1 \%$ dextran sulfate sodium (DSS) for 2 weeks. The disease activity index (DAI), pathological scores and expression of inflammatory cytokines were compared among the groups, and the proportions of fecal bacteria in the colon were analyzed. The body weight and colon length were significantly decreased, and the DAI and pathological scores were significantly increased in the DSS/HFD-treated mice compared with the non-DSS-treated and control diet mice. Regarding the expression of inflammatory cytokines, the levels of interleukin $(I L)-6, I L-1 \beta$ and tumor necrosis factor- $\alpha$ were significantly increased, and the expression of the tight junction protein occludin was significantly decreased in the DSS/HFD-treated mice. The total bacterial count was increased in the HFD mice. Taken together, these results indicate that an HFD resulted in the deterioration of intestinal barrier function and increased susceptibility to DSS-induced colitis.
\end{abstract}

\section{Introduction}

Inflammatory bowel disease (IBD) is a chronic inflammatory disorder of the gastrointestinal tract; its incidence is higher in western countries but is rapidly increasing in Asian popula-

Correspondence to: Dr Shuji Kanmura, Digestive and Lifestyle Diseases, Kagoshima University Graduate School of Medical and Dental Sciences, 8-35-1 Sakuragaoka, Kagoshima 890-8544, Japan E-mail: skanmura@m2.kufm.kagoshima-u.ac.jp

Key words: inflammatory bowel disease, fructose, paracellular permeability, tight junction, microbiota tions (1). Epidemiological and clinical evidence has shown an association between IBD and several environmental factors, antibiotic use, modern lifestyle and diet $(2,3)$. One specific risk factor is the consumption of fructose; fructose intake is reported to cause gut symptoms, and lead not only to the exacerbation of IBD symptoms but also to those of irritable bowel syndrome (4). Previous reports have revealed a link between fructose and the exacerbation of gut symptoms in patients with IBD, and a diet low in fermentable oligo-, di- and mono-saccharides, and polyols may be an effective dietary therapy for patients with IBD and coexisting functional gut symptoms $(5,6)$.

Fructose is an inverted sugar that is used in popular carbonated beverages (7-9). It is absorbed from the intestinal lumen by diffusion, a process that is facilitated by the mucosal GLUT5 transporter protein, a type of glucose-dependent transporter (10). Previous studies demonstrated that increased fructose consumption resulted in the accumulation of lipids in the liver, leading to elevated plasma triglyceride levels and oxidative stress (11-13). Therefore, the excessive intake of fructose is considered a risk factor for lifestyle-related diseases, including obesity, type 2 diabetes mellitus and non-alcoholic fatty liver disease (14-17). It was reported that chronic fructose intake in mice was associated with the loss of occludin in the small intestine, and that gut bacterial overgrowth was associated with the translocation of bacterial endotoxins from the intestine to the liver $(18,19)$.

However, the effects of fructose on the large intestinal tract remain to be fully elucidated, particularly with regard to intestinal inflammation, intestinal barrier function and gut microbiota in the colon. In the present study, a mouse model of colitis was used to analyze the impact of fructose on each of these regions.

\section{Materials and methods}

Animals and diets. Six-week-old male C57BL/6J mice (weight is 20 g; Kyudo Co., Ltd., Kumamoto, Japan) were randomly allocated into four experimental groups: Group $C(n=7)$, the control group, was fed standard chow (SC) and normal water; Group D ( $\mathrm{n}=10)$ was fed SC and water containing 1\% dextran sulfate sodium (DSS; MW 5,000; Wako Pure Chemical 
Industries, Ltd., Osaka, Japan); Group F (n=7) was fed a high-fructose diet (HFD; Oriental Yeast Co., Ltd., Tokyo, Japan) and normal water; and Group FD (n=10) was fed an HFD and water containing $1 \%$ DSS. The mixture containing $1 \%$ DSS and HFD was supplied for 2 weeks, without normal drinking water. The composition of HFD was $60 \%$ fructose, $20.7 \%$ casein, $0.3 \%$ methionine, $5.0 \%$ lard, $9.249 \%$ cellulose, $4.5 \%$ vitamins and minerals, $0.25 \%$ choline bitartrate, and $0.001 \%$ tertiary butylhydroquinone. Each day, the mice were visually assessed to investigate the presence of loose stools, blood in the feces or decreased mobility, the latter considered the disease activity index (DAI) score, to evaluate the clinical activity. The DAI scores were determined based on the following criteria: Changes in body weight, stool consistency and fecal occult blood or visible bleeding. These parameters were assessed on a scale of 0-4 (20-22). The colon tissues were fixed in $10 \%$ formalin, embedded in paraffin, and stained with hematoxylin and eosin to evaluate the pathological activity. The pathological score was used to evaluate the histological activity (23). In addition, a section of the colon tissue was stored at $-80^{\circ} \mathrm{C}$ in order to analyze the gene expression. All animals were housed under standard conditions (in a $24^{\circ} \mathrm{C}$-controlled environment with a 12-h light/dark cycle) and received humane care in compliance with the institutional guidelines. The present study was approved by the institutional Animal Care and Use Committees of Kagoshima University (Kagoshima, Japan) and was performed in accordance with the Committees' guidelines for animal experiments.

ELISA for fecal fructose. The feces of the mice that were fed SC (Group C) and those fed with the HFD (Group F) were collected; $10 \mu \mathrm{g}$ of feces from each group was diluted in $400 \mu \mathrm{l}$ of distilled water and the level of fructose in the feces was measured using an EnzyChrom Fructose Assay kit (cat. no. EFRU-100; BioAssay Systems LLC, Hayward, CA, USA), a Bio-Rad iMark Microplate Reader and the Microplate Manager software program v6 (Bio-Rad Laboratories, Inc., Hercules, CA, USA).

RNA isolation and the gene expression assay. Total RNA $(0.5 \mu \mathrm{g})$ was reverse transcribed using a PrimeScript RT Reagent kit (Takara Bio Inc., Otsu, Japan) at $37^{\circ} \mathrm{C}$ for $15 \mathrm{~min}$ and $85^{\circ} \mathrm{C}$ for $5 \mathrm{sec}$. Each reaction mixture for RT (total volume $20 \mu \mathrm{l}$ ) consisted of total RNA (adjusted to $500 \mathrm{ng}, 2.5 \mu \mathrm{l}$ ), oligo dT PCR primer $(50 \mu \mathrm{M}, 1 \mu \mathrm{l}), 5 \mathrm{X}$ PrimeScript buffer $(4 \mu \mathrm{l})$, PrimeScript RT enzyme mix $(1 \mu \mathrm{l})$, random 6 mers $(100 \mu \mathrm{M}$, $1 \mu \mathrm{l})$ and RNase free distilled $\mathrm{H}_{2} \mathrm{O}(10.5 \mu \mathrm{l})$. The synthesized cDNA was amplified by RT-qPCR using SYBR ${ }^{\circledR}$ Premix Ex Taq ${ }^{\mathrm{TM}}$ II (Takara Bio Inc.). Each reaction mixture for PCR (total volume $20 \mu \mathrm{l}$ ) consisted of cDNA (adjusted to $500 \mathrm{ng}$, $2 \mu \mathrm{l})$, forward and reverse PCR primer $(10 \mu \mathrm{M}, 0.8 \mu \mathrm{l}$ each), SYBR Premix Ex Taq II (10X, $10 \mu \mathrm{l})$, ROX reference dye (50X, $0.4 \mu \mathrm{l})$ and distilled $\mathrm{H}_{2} \mathrm{O}(6 \mu \mathrm{l})$. The cycling conditions were as follows: One cycle at $95^{\circ} \mathrm{C}$ for $30 \mathrm{sec}$ followed by 35 cycles each at $95^{\circ} \mathrm{C}$ for $5 \mathrm{sec}$ and $60^{\circ} \mathrm{C}$ for $34 \mathrm{sec}$. Data were collected and analyzed using the ABI Prism software program v2.3 (Applied Biosystems; Thermo Fisher Scientific, Inc., Waltham, MA, USA). The following primers were used: Mouse interleukin (IL)-1 $\beta, I L-10, I L-6$, transforming growth factor- $\beta$ (TGF- $\beta)$, tumor necrosis factor- $\alpha(T N F-\alpha), G A P D H$, occludin, claudin-1 and zonula occludens-1 ( $\mathrm{O} O-1)$ from Takara Bio Inc., and Sigma-Aldrich; Merck KGaA (Darmstadt, Germany), listed in Table I, and human occludin, claudin-1 and ZO-1 (Table II).

Western blot analysis. The HT-29 cells were exposed to fructose $(0,0.01,0.1,1$ or $10 \mathrm{mM})$, and total protein was extracted from the cells using RIPA buffer (Sigma-Aldrich; Merck KGaA). The cell lysates were analyzed for protein content using a Lowry Assay (Bio-Rad Laboratories, Inc.). Cell lysates containing equal quantities of protein $(10 \mu \mathrm{g})$ from each group were separated on $10 \%$ SDS polyacrylamide gels (Wako Pure Chemical Industries, Ltd.) and electroblotted onto polyvinylidene fluoride membranes. (Bio-Rad Laboratories, Inc.). Following blocking overnight at $4{ }^{\circ} \mathrm{C}$ with $5 \%$ non-fat milk, the blots were probed with primary antibodies for $1 \mathrm{~h}$ at room temperature. The membranes were then incubated with the following primary antibodies: Anti-occludin antibody (1:1,000; cat. no. 71-1500; Invitrogen; Thermo Fisher Scientific, Inc.), anti-claudin 1 antibody (1:1,000; cat. no. ab15098; Abcam, Cambridge, MA, USA), anti-ZO-1 antibody (1:1,000; cat. no. 40-2200; Thermo Fisher Scientific, Inc.), and $\beta$-actin antibody (1:1,000; cat. no. 4970; Cell signaling Technology, Inc., Danvers, MA, USA). The membranes were washed with Tris buffered saline (Bio-Rad Laboratories, Inc.) with 0.1\% Tween-20 (Wako Pure Chemical Industries, Ltd.) three times. Following incubation with horseradish peroxidase-conjugated secondary antibodies $(1: 1,000$; cat. no. 7074; Cell Signaling Technology, Inc.) for $1 \mathrm{~h}$ at room temperature, the membrane was visualized for the reaction using electrogenerated chemiluminescence western blotting detection reagents (GE Healthcare Life Sciences, Shanghai, China). Expression level quantification was performed using Image J v1.48 (National Institutes of Health, Bethesda, MD, USA), and $\beta$-actin was used as an internal control.

Extraction of DNA from fecal samples and the determination of the intestinal bacteria composition. DNA was extracted from fecal samples and standard strains using ISOFECAL for Beads Beating (Nippon Gene Co., Ltd., Tokyo, Japan) for quantification by RT-qPCR analysis. First, $20 \mu \mathrm{g}$ of feces was diluted in $200 \mu \mathrm{l}$ of distilled water. The standard strains were used for each group: Bifidobacterium longum JCM 1217 for the Bifidobacterium group, 06TCa19 for the Lactobacillus group, Clostridium coccoides JCM 1395 for the C. coccoides group, and Bacteroides fragilis JCM 11019 for the Bacteroides-Prevotella group. The Bifidobacterium subspecies and the C.coccoides, Bacteroides-Prevotella and Lactobacillus groups were detected using SYBR-Green RT-qPCR analysis. The following primers were used (Sigma-Aldrich; Merck $\mathrm{KGaA}$ ): Bifidobacterium longum, Lactobacillus, $C$. coccoides, Bacteroides fragilis (Table III). Gene quantification was performed using an ABI Prism 7700 sequence detection system (Applied Biosystems; Thermo Fisher Scientific, Inc.). To determine the total bacteria count, fecal samples were stained with 4',6-diamidino-2-phenylindole solution (Dojindo Molecular Technologies, Inc., Kumamoto, Japan), and the bacteria were counted using a hemocytometer for bacteria (AS ONE Corporation, Osaka, Japan) under a fluorescence microscope (Carl Zeiss AG, Oberkochen, Germany). 
Table I. Primers for reverse transcription polymerase chain reaction analysis for mice.

\begin{tabular}{lll}
\hline Gene & \multicolumn{1}{c}{ Forward $\left(5^{\prime}-3^{\prime}\right)$} & \multicolumn{1}{c}{ Reverse $\left(5^{\prime}-3^{\prime}\right)$} \\
\hline$I L-1 \beta$ & TCCAGGATGAGGACATGAGCAC & GAACGTCACACACCAGCAGGTTA \\
$I L-10$ & GCCAGAGCCACATGCTCCTA & GATAAGGCTTGGCAACCCAAGTAA \\
$I L-6$ & CCACTTCACAAGTCGGAGGCTTA & CCAGTTTGGTAGCATCCATCATTTC \\
$T G F-\beta$ & GTGTGGAGCAACATGTGGAACTCTA & TTGGTTCAGCCACTGCCGTA \\
$T N F-\alpha$ & TATGGCCCAGACCCTCACA & GGAGTAGACAAGGTACAACCCAT \\
Occludin & GAGTTAACGTCGTGGACCGGTATC \\
$Z \mathrm{-}-1$ & CGGTTCATGGCCTCTGAATCTATAA & CTGCCTTAGCAGTTTCAGTTTGGAG \\
Claudin- 1 & CTTAATTAGTGAGCTTGGGCAAAGG & CATTTCTGAAACCATCAAGTCCACA \\
$G A P D H$ & TGTGTCCGTCGTGGATCTGA & CTCATGCAACATAGGCAGGACA \\
\hline
\end{tabular}

$I L$, interleukin; $T G F-\beta$, transforming growth factor- $\beta$; TNF- $\alpha$, tumor necrosis factor- $\alpha$; ZO- 1 , zonula occludens- 1 ; GAPDH, glyceraldehyde 3-phosphate dehydrogenase.

Table II. Primers for reverse transcription-polymerase chain reaction analysis for human cells.

\begin{tabular}{lll}
\hline Gene & \multicolumn{1}{c}{ Forward 5'-3' } & \multicolumn{1}{c}{ Reverse 5'-3' } \\
\hline Occludin & TCCAATGGCAAAGTGAATGA & GCAGGTGCTCTTTTTGAAGG \\
$Z O-1$ & TGAGGCAGCTCACATAATGC & GGTCTCTGCTGGCTTGTTTC \\
Claudin-1 & CCGTTGGCATGAAGTGTATG & CCAGTGAAGAGAGCCTGACC \\
GAPD & GAGTCAACGGATTTGGTCGT & TTGATTTTGGAGGGATCTCG \\
\hline
\end{tabular}

ZO-1, zonula occludens-1; GAPDH, glyceraldehyde 3-phosphate dehydrogenase.

Table III. Primers for reverse transcription polymerase chain reaction analysis for bacteria.

\begin{tabular}{lll}
\hline Gene & Forward 5'-3' & Reverse 5'-3' \\
\hline Lactobacillus group & AGCAGTAGGGAATCTTCCA & CACCGCTACACATGGAG \\
Bifidobacterium group & TCGCGTCYGGTGTGAAAG & CCACATCCAGCRTCCAC \\
C. coccoides & AAATGACGGTACCTGACTAA & CTTTGAGTTTCATTCTTGCGAA \\
Bacteroides-Prevotella group & GAAGGTCCCCCACATTG & CAATCGGAGTTCTTCGTG \\
\hline
\end{tabular}

Cell culture. The human HT-29 colon cancer cell line (European Collection of Cell Cultures, no. EC910722201) was obtained from Dainippon Sumitomo Pharma Biomedical (Osaka, Japan) and maintained at $37^{\circ} \mathrm{C}$ in $5 \% \mathrm{CO}_{2}$ and McCoy's 5A medium supplemented with $10 \%$ heat-inactivated fetal bovine serum (Gibco; Thermo Fisher Scientific, Inc.), streptomycin, and penicillin.

Paracellular permeability assay. The epithelial permeability to macromolecules was determined using fluorescein isothiocyanate (FITC)-dextran. The $1.5 \times 10^{5}$ cells/well of HT-29 cells were seeded on the upper chamber with $8-\mu \mathrm{m}$ pores and cultured until they reached full confluence in monolayers. The cells in the upper chamber were exposed to fructose $(0.001$, $0.01,0.1,1$ or $10 \mathrm{mM})$ and then incubated with FITC-dextran $(5 \mathrm{mg} / \mathrm{ml})$ for $24 \mathrm{~h}$ at $37^{\circ} \mathrm{C}$ in a $\mathrm{CO}_{2}$ incubator. The fluores- cence of FITC-dextran in the lower chambers was sequentially measured following exposure to fructose $(0,0.5$ and $1 \mathrm{~h}) 24 \mathrm{~h}$ at $37^{\circ} \mathrm{C}$ in a $\mathrm{CO}_{2}$ incubator. The data were calculated as follows: Apparent permeability $(\mathrm{cm} / \mathrm{s})=$ (rate of change in cumulative mass of FITC transferred to lower chamber)/[(surface area of cell culture insert) $\mathrm{x}$ (initial concentrations of radio-labeled substances)] (24). This assay was conducted five times.

LDH assay. An LDH Cytotoxicity Detection kit was used (Takara Bio, Inc.) to examine the cytotoxicity of fructose towards HT-29 cells that were exposed to various concentrations of fructose $(0,0.001,0.01,0.1,1$ or $10 \mathrm{mM})$ for $0.5,1$ or $2 \mathrm{~h}$. An ELISA was performed using a Bio-Rad iMark Microplate Reader and the Microplate Manager software program (Bio-Rad Laboratories, Inc.). This LDH assay was conducted five times. 
Statistical analysis. All data are expressed as the mean \pm standard error of the mean. Differences between two groups were analyzed using the Mann-Whitney U test. Differences among multiple groups were compared using the Kruskal-Wallis test and one-way analysis of variance (IBM SPSS v23.0; IBM Corp., Armonk, NY, USA). $\mathrm{P}<0.05$ was considered to indicate a statistically significant difference.

\section{Results}

Fecal concentrations of fructose in mice. First, the fecal concentrations of fructose were measured and the results of Groups $\mathrm{C}$ and $\mathrm{F}$ were compared using an ELISA following 14 days of feeding with normal chow and an HFD, respectively. The median concentrations of fructose in Groups $\mathrm{C}$ and $\mathrm{F}$ were 864 and 1,679 $\mu \mathrm{M}$, respectively. The level of fructose in the stools of Group F was significantly higher than that in Group C (Fig. 1).

HFD affects body weight and aggravates DSS-induced colitis. No significant difference in the rates of body weight change were found among Groups C, D and F; however, the rate of body weight change in Group $\mathrm{F}$ tended to be lower compared with those in Groups C and D. The rate of body weight change in Group FD was significantly decreased compared with that in the other groups on day 14 (Fig. 2A). The colon length in Group FD was significantly shorter than in the other groups (Fig. 2B). In addition, the pathological and DAI scores of Group FD were significantly increased compared with those of Groups C, D and F; furthermore, the scores of Group $\mathrm{F}$ were significantly increased compared with those of Group C (Fig. 2C and D). On pathological examination of the colon, mild inflammatory cell infiltration was observed in the mucosal layer, whereas the epithelial cells were normal in Group F. In Group FD, the mucosa was broken by erosion or ulcers, and infiltration of inflammatory cells into the submucosal layer was observed (Fig. 2E).

Excessive intake of fructose induces the expression of inflammatory cytokines in mice. The gene expression levels of $T N F-\alpha, I L-6$ and $I L-1 \beta$ in Groups D and FD were significantly increased compared with those in Group $C$. The gene expression levels of $I L-6$ and $I L-1 \beta$ in Group FD were significantly increased compared with those in Group D; however, the expression of TNF- $\alpha$ in Groups D and FD did not differ to a statistically significant extent. The gene expression levels of $I L-10$ and $T G F-\beta$ in Group FD were significantly increased compared with those in Group D (Fig. 3).

Impact of an excessive fructose intake on the tight-junctions of the mouse intestine. The expression of occludin in Group $\mathrm{F}$ was decreased compared with that in Group C. The expression of occludin in Group FD was significantly decreased compared with that in Group C. Otherwise, the expression levels of $Z O-1$ and claudin-1 in the large intestine of these groups did not differ to a statistically significant extent (Fig. 4).

Excessive fructose intake alters the numbers and proportions of intestinal bacteria. The numbers of intestinal bacteria, including the Lactobacillus group, Bifidobacterium group,

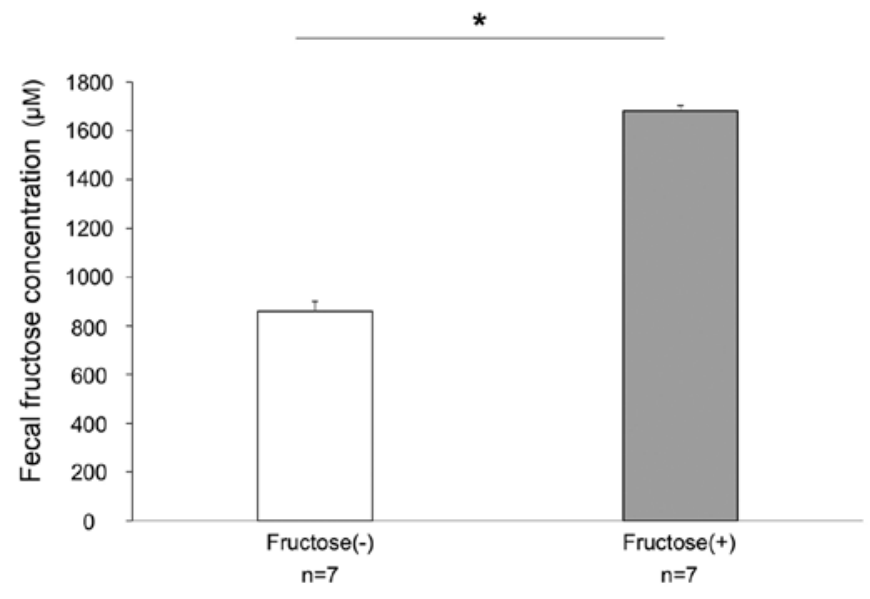

Figure 1. Fecal concentrations of fructose. The data shown are presented as the mean \pm standard error of the mean $(n=7)$. The fecal fructose level in Group F was significantly increased compared with that in Group C. " $\mathrm{P}<0.01$ (Mann-Whitney U test).

C. coccoides group and Bacteroides-Prevotella in each of the groups were measured. The total numbers of bacteria in the colon of Group F and FD were increased compared with those of Groups $\mathrm{C}$ and $\mathrm{D}$. The total counts in each group were as follows: Group C, $4.3 \times 10^{10} \mathrm{CFU} / \mathrm{ml}$; Group D, $4.4 \times 10^{10} \mathrm{CFU} / \mathrm{ml}$; Group F,


The counts (and percentages) of Bacteroides-Prevotella in the four groups were as follows: Group C, $2.3 \times 10^{8}$

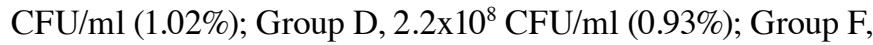
$6.1 \times 10^{8} \mathrm{CFU} / \mathrm{ml}$ (1.59\%); and Group FD, 6.2×10 ${ }^{8} \mathrm{CFU} / \mathrm{ml}$ $(1.90 \%)$. The counts of $C$. coccoides were as follows: Group C, $3.0 \times 10^{9} \mathrm{CFU} / \mathrm{ml}$ (13.0\%); Group D, 5.2×109 $\mathrm{CFU} / \mathrm{ml}$ (21.48\%); Group F, $1.1 \times 10^{10} \mathrm{CFU} / \mathrm{ml}$ (28.9\%); and Group FD, $7.7 \times 10^{9} \mathrm{CFU} / \mathrm{ml}$ (23.72\%). The counts of the Lactobacillus group were as follows: Group C, $5.7 \times 10^{8} \mathrm{CFU} / \mathrm{ml}(2.5 \%)$;

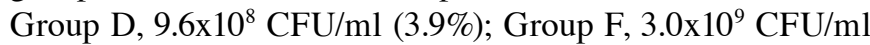
(7.9\%); and Group FD, 4.1 $\times 10^{9} \mathrm{CFU} / \mathrm{ml}$ (12.7\%). Finally, the counts of the Bifidobacterium group were as follows: Group C, $2.0 \times 10^{7}(0.08 \%)$; Group D, $1.0 \times 10^{7}(0.04 \%)$; Group F, $4.1 \times 10^{7}(0.1 \%)$; and Group FD, $4.9 \times 10^{7}(0.15 \%)$. Excessive fructose intake affected the numbers and percentages of the Bacteroides-Prevotella group, C. coccoides, the Lactobacillus group and the Bifidobacterium group (Fig. 5B).

Fructose decreases the expression of occludin in the HT-29 colon cell line. The gene expression levels of occludin, $\mathrm{ZO}-1$ and claudin-1 of HT-29 cells following exposure to several concentrations of fructose were measured using RT-qPCR analysis, and the gene expression of occludin was significantly decreased by fructose exposure in a dose-dependent manner. The gene expression of ZO-1 and claudin-1 did not differ in vitro (Fig. 6A). The expression of tight junction proteins was also examined by western blotting. The expression of occludin in HT-29 cells was significantly decreased by fructose exposure in a dose-dependent manner, whereas no differences in the expression ZO-1 or claudin-1 were observed (Fig. 6B and C).

Fructose enhances paracellular permeability and cytotoxicity in the HT-29 colon cell line. The effect of fructose on 
A

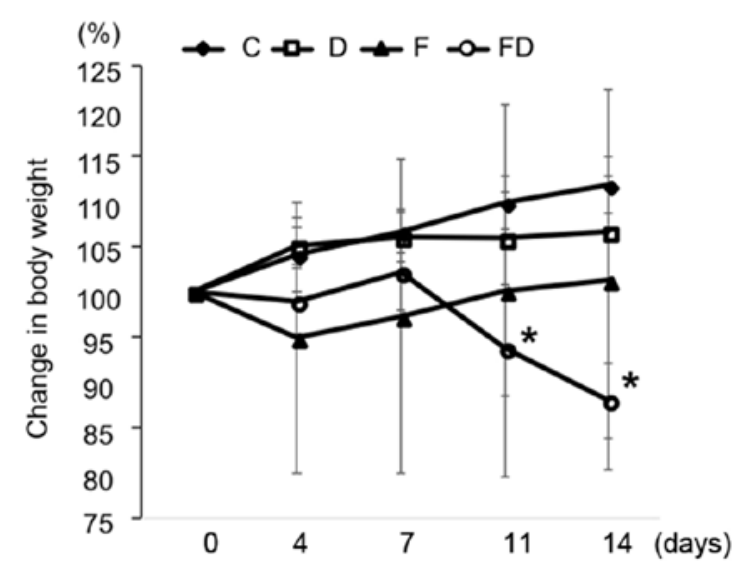

C

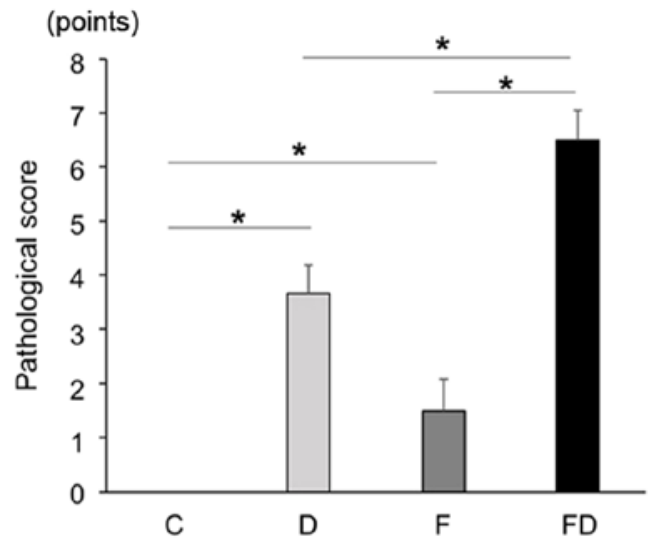

B



D

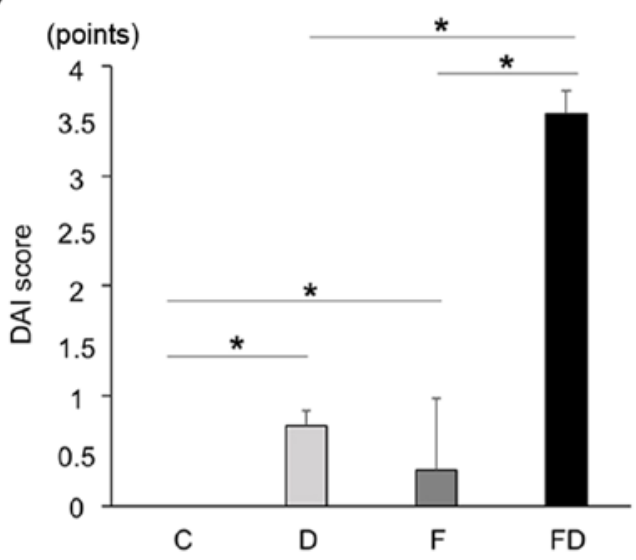

E

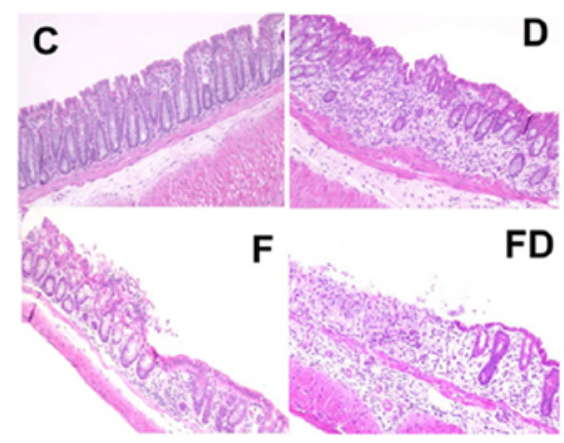

Figure 2. Mice were allocated into four groups: Standard chow and normal water (Group C, $n=7$ ); standard chow and water containing DSS (Group D, $n=10)$; a high fructose diet + normal water (Group F, n=7); and a high fructose diet + water containing DSS (Group FD, n=10). " $\mathrm{P}<0.01$. (A) Rate of body weight change. The body weight of Group FD was significantly decreased compared with that of Group D ("P<0.01). (B) Colon length of each group. The colon of Group FD was significantly shorter compared with the other groups. (C) Pathological scores of each group. The scores of Groups F and FD were significantly increased compared with that of Group C. (D) DAI scores of each group. The scores of Groups F and FD were significantly increased compared with that of Group C. (E) Pathological examinations of the colon in each group. In Group F, mild inflammatory cell infiltration was observed in the mucosal layer, although the epithelial cell was normal. Erosion or ulcers, and infiltration of inflammatory cells into the submucosal layer was observed in Groups D and FD. In particular, severe intestinal inflammation was observed in Group FD. Kruskal-Wallis and one-way analysis of variance was used for statistical analysis. DAI, disease activity index; H\&E, hematoxylin and eosin.

paracellular permeability to macromolecules was examined. The HT-29 cells were exposed to various concentrations of fructose $(0,0.001,0.01,0.1,1$ or $10 \mathrm{mM})$ for several sequential periods $(0,0.5$ or $1 \mathrm{~h})$. Fructose enhanced the paracellular permeability of the cells in a concentration- and time-dependent manner (Fig. 7A). Furthermore, the cytotoxicity of fructose to 

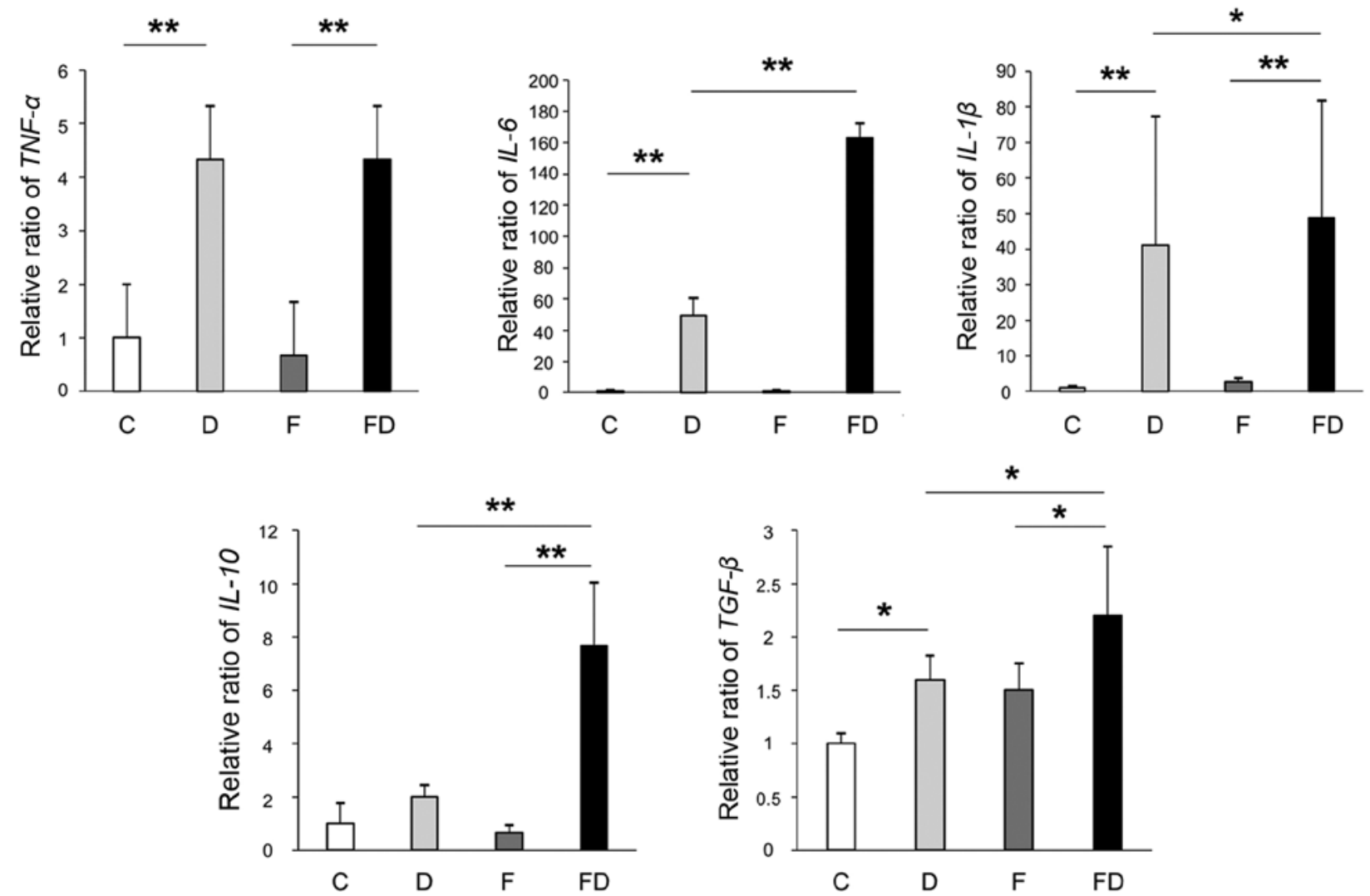

Figure 3. mRNAs of inflammatory and anti-inflammatory cytokines were measured by reverse transcription-quantitative polymerase chain reaction analysis ${ }^{*} \mathrm{P}<0.05$ and ${ }^{* *} \mathrm{P}<0.01$ (Kruskal-Wallis and one-way analysis of variance). The levels of $T N F-\alpha, I L-6$, and $I L-1 \beta$ in Groups D and FD were significantly increased compared with those in Group C. $T N F-\alpha$ in Groups D and FD did not differ to a statistically significant extent. The levels of $I L-6, I L-1 \beta$ and $I L-10$ in Group FD were significantly increased compared with those in Group D. The level of $T G F-\beta$ was also significantly increased. TNF- $\alpha$, tumor necrosis factor- $\alpha$; $I L$, interleukin; $T G F-\beta$, transforming growth factor- $\beta$.
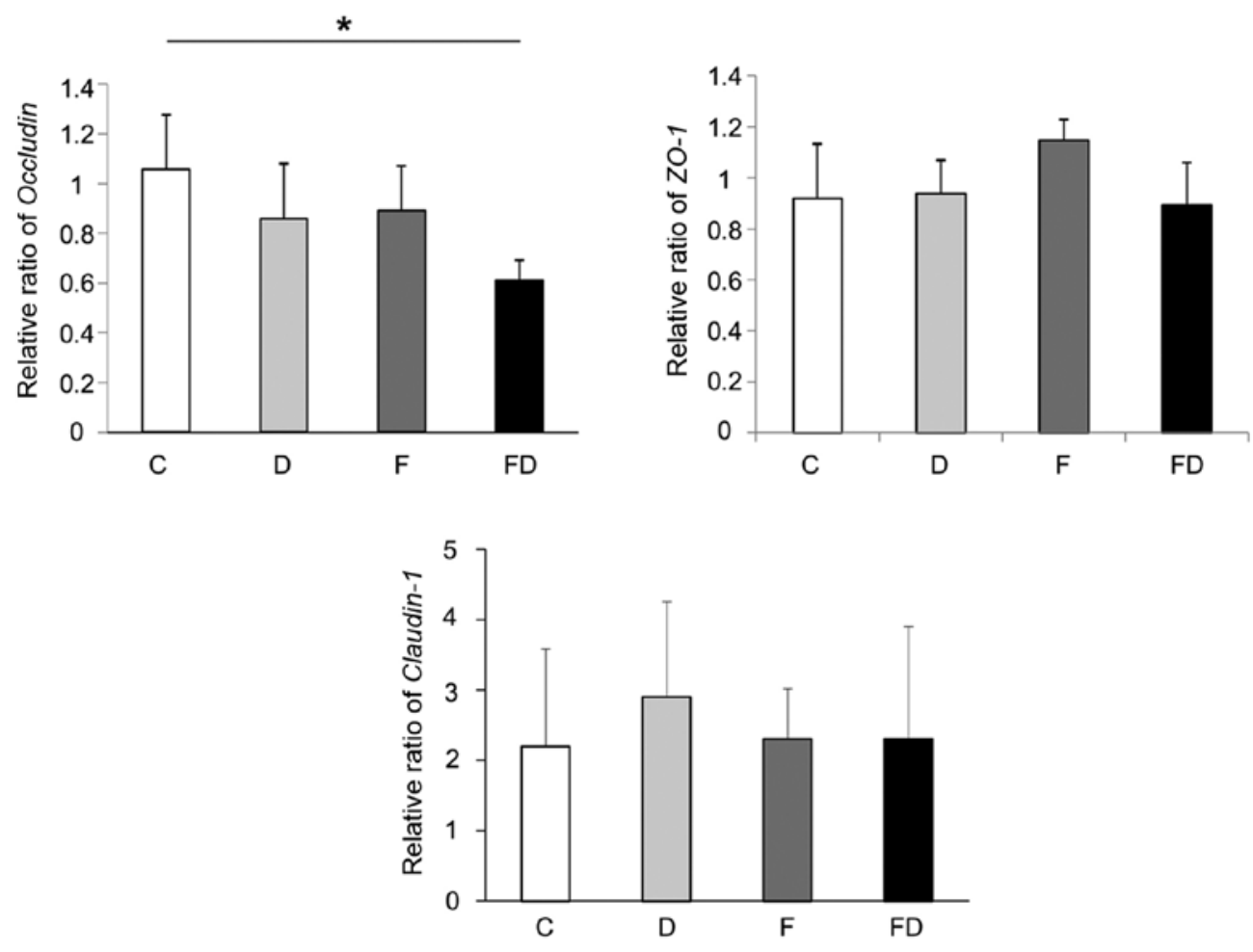

Figure 4. mRNAs of tight junction proteins were measured by reverse transcription-quantitative polymerase chain reaction analysis and analyzed using Kruskal-Wallis and one-way analysis of variance. The gene expression of occludin in Group FD was significantly decreased compared with that in Group C. ${ }^{\prime} \mathrm{P}<0.01$. The expression of occludin in Group D and Group F tended to be decreased compared with that in Group C. The levels of ZO-1 and claudin-1 did not differ to a statistically significant extent among the groups. ZO-1, zonula occludens-1. 
A

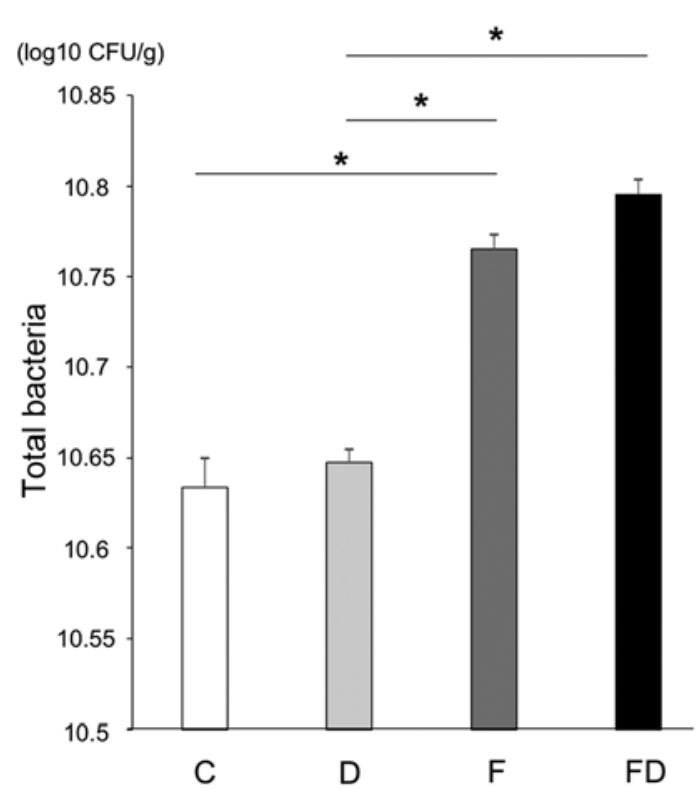

B

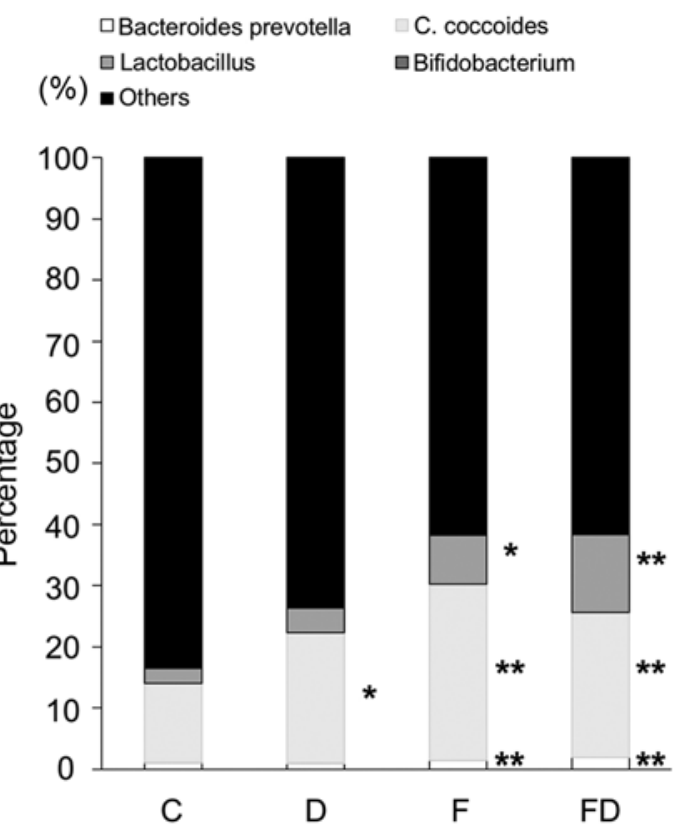

Figure 5. Bacterial numbers. Bacteria in the feces of each groups were measured by a 16s rRNA analysis, and the proportions of the Lactobacillus group, Bifidobacterium group, C. coccoides and Bacteroides-Prevotella group were measured. * $\mathrm{P}<0.01$. (A) Numbers of bacteria were significantly increased in Groups F and FD. (B) Percentages of the Bacteroides-Prevotella group, the Lactobacillus group and C. coccoides were marginally increased in Groups F and FD. Compared with Group $\mathrm{C}$. ${ }^{*} \mathrm{P}<0.05$ and ${ }^{* *} \mathrm{P}<0.01$ (Kruskal-Wallis and one-way analysis of variance).

cells was examined. The HT-29 cells were exposed to various concentrations of fructose $(0,0.001,0.01,0.1,1$ or $10 \mathrm{mM})$ for several periods $(0.5,1$ or $2 \mathrm{~h})$. Fructose injured epithelial cells in a concentration- and time-dependent manner (Fig.7B).

\section{Discussion}

The present study showed that excess fructose intake was cytotoxic in epithelial cells; it reduced the expression of occludin, a tight junction protein, and impaired intestinal barrier function, thereby inducing a leaky gut. Furthermore, the overconsumption of fructose induced the overgrowth of intestinal bacteria, which was considered to eventually exacerbate colitis in the mouse model. The present study is the first, to the best of our knowledge, to reveal the mechanism underlying the impact of fructose on intestinal inflammation and intestinal barrier function in the colon.

It was found that fructose increased sensitivity to DSS, thereby aggravating intestinal inflammation. In order to investigate the influence of fructose on epithelial cell function and DSS susceptibility, a colitis model was used in which mice were administered low concentrations of DSS, which causes relatively minimal damage to the epithelium. It was shown that, under conditions of excessive fructose consumption, fructose reached the colon and affected the intestinal barrier function of epithelial cells, resulting in increased paracellular permeability. In the in vivo assays, the pathology and DAI scores in the fructose intake group (Group F) were significantly increased compared with those in the control group. Group F demonstrated mild inflammatory cell infiltration but no increased secretion of $T N F-\alpha$, $I L-6$ or $I L-1 \beta$ in the colon tissue. These results suggested that the number of infiltrating inflammatory cells was rela- tively small, and as whole colon tissue was used for gene expression analysis, the difference in cytokine expression was not significant between Groups $\mathrm{C}$ and $\mathrm{F}$. The in vitro assays revealed that fructose exposure increased epithelial permeability in a time- and concentration-dependent manner. Therefore, the intake of fructose was associated with the development of a leaky gut, which resulted in the movement of bacterial antigens (e.g., lipopolysaccharides) into the submucosa, leading to the development of intestinal inflammation. Furthermore, it has been reported that fructose increases the activation of intestinal macrophages, which secrete cytokines (25). Therefore, the excessive intake of fructose may affect intestinal macrophages, although further investigations are required.

The present study revealed the impact of fructose on epithelial barrier function in the large intestine. Fructose decreased the expression of the gene coding for the tight junction protein occludin and the protein itself, whereas the expression levels of $Z O-1$ and claudin-1 were unchanged. A previous report demonstrated that bacterial products, including ethanol and acetaldehyde, may cause the loss of occludin and increase permeability of the intestine in a mouse model of fructose-induced hepatic steatosis (26). Another report noted that the intake of fructose was associated with a marked loss of the tight junction proteins occludin and $Z O-1$ in the duodenum $(27,28)$. However, the reason why fructose exposure decreased only small and large intestinal occludin expression remains to be elucidated. Further investigations are required to address this question.

Although there was a marginal increase in the fecal concentration of fructose in the normal diet group (Group C; Fig. 1), there was no effect on the colonic epithelium. The main ingredient in the chow fed to the normal diet group was corn; 
A
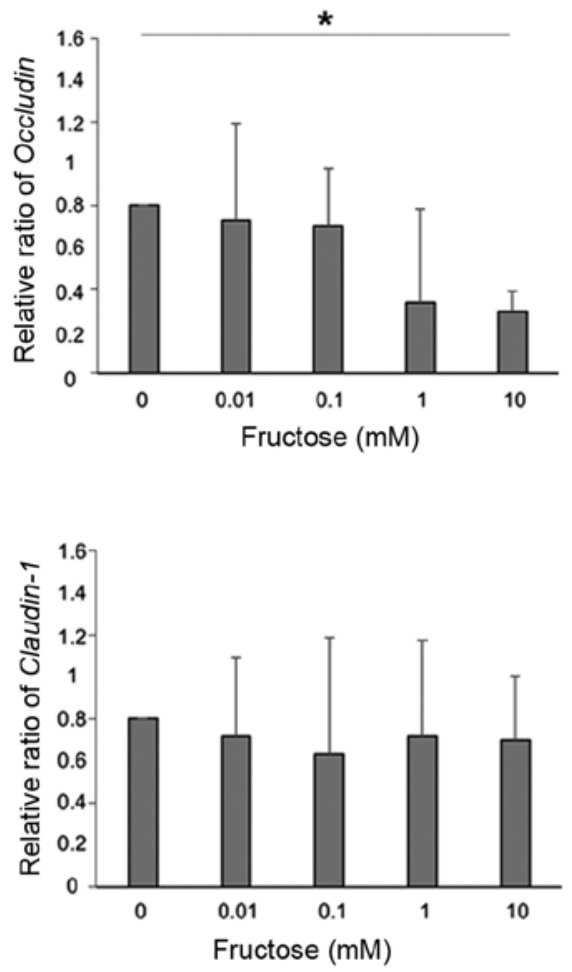

C

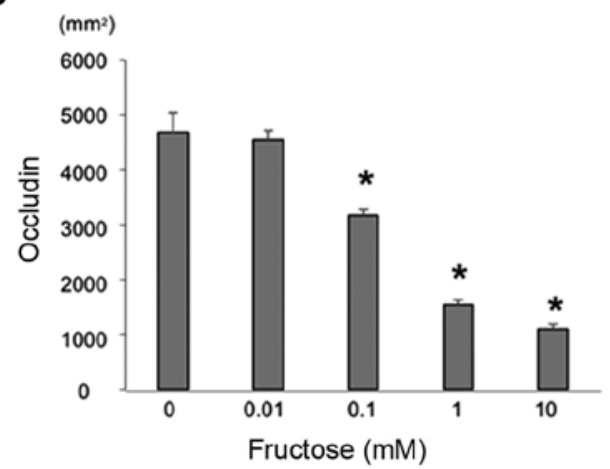

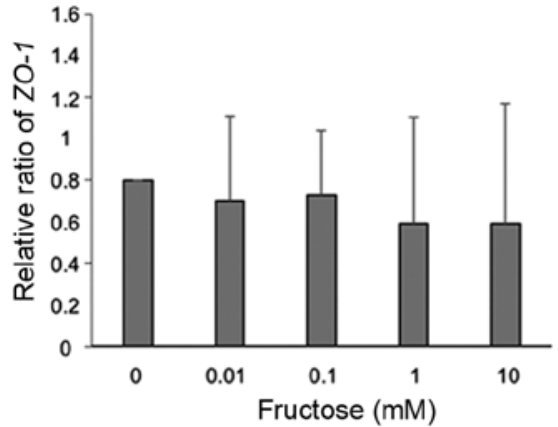

B
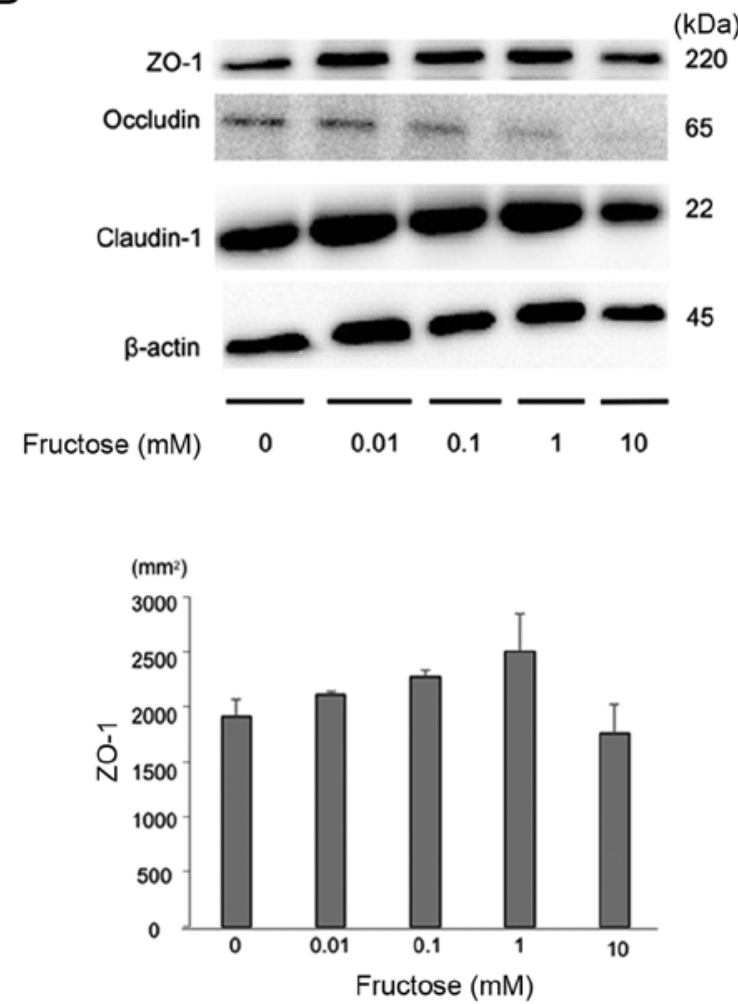

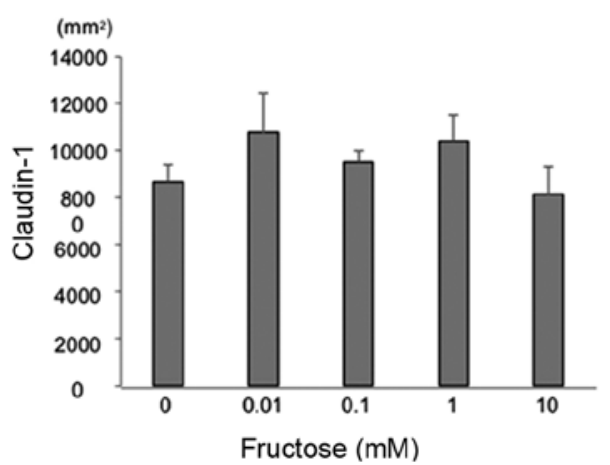

Figure 6. Gene and protein expression levels of tight junction proteins. (A) Ratio of occludin mRNA was significantly decreased by fructose in a concentration-dependent manner. The levels of $\mathrm{ZO}-1$ and claudin-1 were not decreased. (B) Protein expression of tight junction proteins examined using western blotting. (C) Expression level quantification was performed using Image $\mathrm{J}$ v1.48 and $\beta$-actin was used as an internal control. The protein expression of occludin was significantly decreased by fructose in a concentration-dependent manner. However, the protein expression levels of $Z O-1$ and claudin- 1 were not decreased. ${ }^{\mathrm{P}}<0.01$ vs. $0 \mathrm{mM}$ (Kruskal-Wallis and one-way analysis of variance). ZO-1, zonula occludens-1.

therefore, following consumption, it was metabolized to fructose. However, the fecal fructose concentration in Group $\mathrm{C}$ was within normal levels and not be expected to induce intestinal inflammation.
A previous study demonstrated that fructose had an impact on bacterial overgrowth (29). The present study confirmed that excessive fructose intake increased the numbers of certain intestinal bacteria in the colon. In particular, the ratio of the 
A

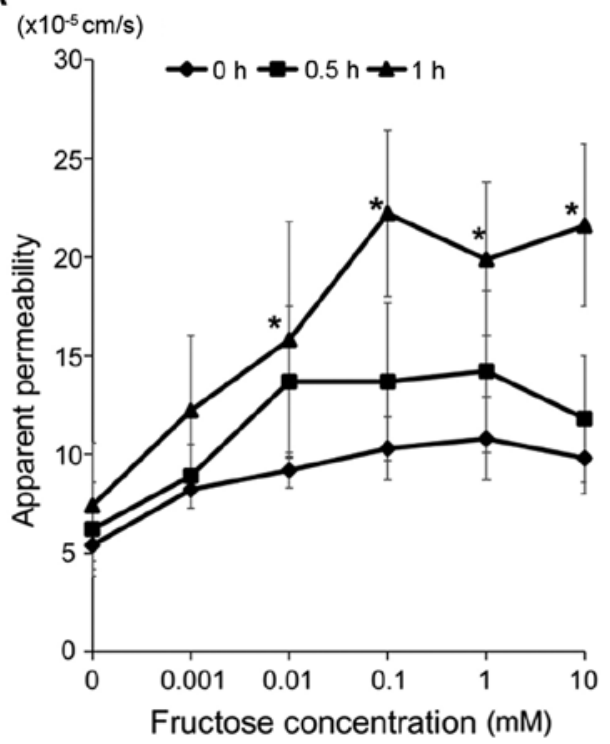

B

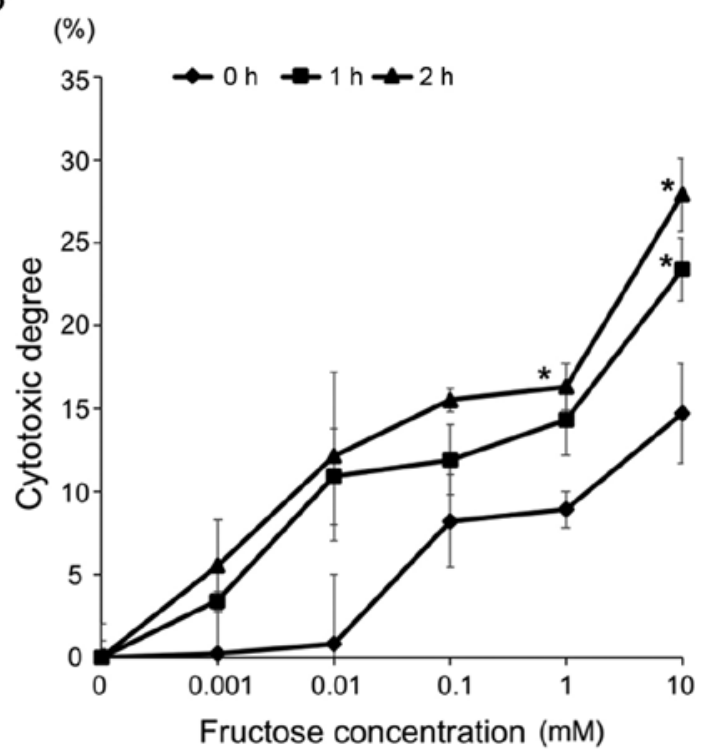

Figure 7. Effect of fructose on paracellular permeability and the cytotoxicity of fructose to cells. The different concentrations of fructose were compared to $0 \mathrm{mM}$. "P<0.01; Kruskal-Wallis and one-way analysis of variance. (A) Fructose enhanced the paracellular permeability of the cells in a concentration- and time-dependent manner. (B) Fructose injured the cells in a concentration- and time-dependent manner.

Bacteroides-Prevotella group was elevated in the mice that were fed the HFD (Fig. 5A and B). Bacteroides is the leading cause of anaerobic bacteremia and sepsis, and the toxin of this genus contributes to the development of colitis and intestinal malignancy (30). In addition, a previous study showed that the Bacteroides group was demonstrated to cause intestinal inflammation and promoted intestinal permeability in patients with IBD (31). For these reasons, the present study focused on the Bacteroides group. A previous study demonstrated a lower proportion of Bacteroides species in mice fed an HFD (32), whereas others demonstrated a marked increase in Bacteroides species in mice fed a standard diet plus high-fructose syrup (33). It is possible that the composition of Bacteroides species is affected by the method of fructose administration, for example, liquid vs. solid formulations. However, further investigation is required to fully understand the reasons for alterations in Bacteroides species.

The present study had several limitations. First, the microbiota were analyzed using RT-qPCR, which was able to detect the numbers of only four intestinal bacterial species. To examine the actual influence of fructose on intestinal bacteria, a metagenomic analysis is required. Second, human subjects with IBD who consume large quantities of fructose were not analyzed. Future investigations aim to analyze colon epithelial tight junctions, inflammatory cytokines and microbiota in this population.

In conclusion, the present study demonstrated that fructose increased paracellular permeability and was involved in the overgrowth of intestinal bacteria, thereby inducing intestinal inflammation. The excessive intake of fructose caused leaky gut syndrome, exacerbated enteric inflammation, and was considered to result in the symptoms of IBD.

\section{Acknowledgements}

Not applicable.

\section{Funding}

The present study was supported by Grants-in-Aid for Scientific Research from the Ministry of Education, Culture, Sports, Science and Technology of Japan; and by Grants-in-Aid from the Ministry of Health, Labour and Welfare of Japan (grant no. 26870459).

\section{Availability of data and materials}

All data generated or analyzed during the present study are included in this published article.

\section{Authors' contributions}

Performed the experiments: KK, SK, YM, AT, TM, TF, SA, ST and SH. Analyzed the data: KK, SK and AI. Contributed reagents/materials/analysis tools: KK, SK, FS and YN. Wrote the manuscript: KK, SK, AI. Conceived and designed the experiments: SK and AI.

\section{Ethics approval and consent to participate}

The present study was approved by the institutional Animal Care and Use Committees of Kagoshima University, and was performed in accordance with the Committees' guidelines for animal experiments.

\section{Patient consent for publication}

Not applicable.

\section{Competing interests}

The authors declare no conflicts of interest in association with the present study. 


\section{References}

1. Asakura K, Nishiwaki Y, Inoue N, Hibi T, Watanabe M and Takebayashi T: Prevalence of ulcerative colitis and Crohn's disease in Japan. J Gastroenterol 44: 659-665, 2009.

2. Podolsky DK: Inflammatory bowel disease (1). N Engl J Med 325: 928-937, 1991.

3. Goldsmith JR and Sartor RB: The role of diet on intestinal microbiota metabolism: Downstream impacts on host immune function and health, and therapeutic implications. J Gastroenterol 49: 785-798, 2014.

4. Simrén M and Stotzer PO: Use and abuse of hydrogen breath tests. Gut 55: 297-303, 2006.

5. Gearry RB, Irving PM, Barrett JS, Nathan DM, Shepherd SJ and Gibson PR: Reduction of dietary poorly absorbed short-chain carbohydrates (FODMAPs) improves abdominal symptoms in patients with inflammatory bowel disease-a pilot study. J Crohn's Colitis 3: 8-14, 2009.

6. Li YC and Hsieh CC: Lactoferrin dampens high-fructose corn syrup-induced hepatic manifestations of the metabolic syndrome in a murine model. PLoS One 9: e97341, 2014.

7. Assy N, Nasser G, Kamayse I, Nseir W, Beniashvili Z, Djibre A and Grosovski M: Soft drink consumption linked with fatty liver in the absence of traditional risk factors. Can J Gastroenterol 22 811-816, 2008.

8. Walker RW, Dumke KA and Goran MI: Fructose content in popular beverages made with and without high-fructose corn syrup. Nutrition 30: 928-935, 2014.

9. Jones HF, Butler RN and Brooks DA: Intestinal fructose transport and malabsorption in humans. Am J Physiol Gastrointest Liver Physiol 300: G202-G206, 2011.

10. Arkan MC, Hevener AL, Greten FR, Maeda S, Li ZW, Long JM, Wynshaw-Boris A, Poli G, Olefsky J and Karin M: IKK-beta links inflammation to obesity-induced insulin resistance. Nat Med 11: 191-198, 2005.

11. Diehl AM, Li ZP, Lin HZ and Yang SQ: Cytokines and the pathogenesis of non-alcoholic steatohepatitis. Gut 54: 303-306, 2005.

12. Shi H, Kokoeva MV, Inouye K, Tzameli I, Yin H and Flier JS TLR4 links innate immunity and fatty acid-induced insulin resistance. J Clin Invest 116: 3015-3025, 2006.

13. Gäbele E, Dostert K, Hofmann C, Wiest R, Schölmerich J, Hellerbrand C and Obermeier F: DSS induced colitis increases portal LPS levels and enhances hepatic inflammation and fibrogenesis in experimental NASH. J Hepatol 55: 1391-1399, 2011.

14. Lírio LM, Forechi L, Zanardo TC, Batista HM, Meira EF, Nogueira BV, Mill JG and Baldo MP: Chronic fructose intake accelerates non-alcoholic fatty liver disease in the presence of essential hypertension. J Diabetes Complications 30: 85-92, 2016.

15. Mohammed A, Koorbanally NA and Islam MS: Ethyl acetate fraction of Aframomum melegueta fruit ameliorates pancreatic $\beta$-cell dysfunction and major diabetes-related parameters in a type 2 diabetes model of rats. J Ethnopharmacol 175: 518-527, 2015.

16. Malik VS and Hu FB: Fructose and cardiometabolic health: What the evidence from sugar-sweetened beverages tells us. J Am Coll Cardiol 66: 1615-1624, 2015.

17. Tanoue S, Uto H, Kumamoto R, Arima S, Hashimoto S, Nasu Y, Takami Y, Moriuchi A, Sakiyama T, Oketani M, et al: Liver regeneration after partial hepatectomy in rat is more impaired in a steatotic liver induced by dietary fructose compared to dietary fat. Biochem Biophys Res Commun 407: 163-168, 2011.

18. Wigg AJ, Roberts-Thomson IC, Dymock RB, McCarthy PJ, Grose RH and Cummins AG: The role of small intestinal bacterial overgrowth, intestinal permeability, endotoxaemia, and tumour necrosis factor alpha in the pathogenesis of non-alcoholic steatohepatitis. Gut 48: 206-211, 2001.
19. Brun P, Castagliuolo I, Floreani AR, Buda A, Blasone L, Palù G and Martines D: Increased risk of NASH in patients carrying the $\mathrm{C}(-159) \mathrm{T}$ polymorphism in the $\mathrm{CD} 14$ gene promoter region. Gut 55: 1212, 2006.

20. Jin Y, Lin Y, Lin L, Sun Y and Zheng C: Carcinoembryonic antigen related cellular adhesion molecule 1 alleviates dextran sulfate sodium-induced ulcerative colitis in mice. Life Sci 149: 120-128, 2016.

21. Barnett MP, Dommels YE, Butts CA, Zhu S, McNabb WC and Roy NC: Inoculation with enterococci does not affect colon inflammation in the multi-drug resistance 1a-deficient mouse model of IBD. BMC Gastroenterol 16: 31, 2016.

22. Raup-Konsavage WM, Cooper TK and Yochum GS: A role for MYC in lithium-stimulated repair of the colonic epithelium after DSS-induced damage in mice. Dig Dis Sci 61: 410-422, 2016

23. Park JS, Yi TG, Park JM, Han YM, Kim JH, Shin DH, Tak SJ, Lee K, Lee YS, Jeon MS, et al: Therapeutic effects of mouse bone marrow-derived clonal mesenchymal stem cells in a mouse model of inflammatory bowel disease. J Clin Biochem Nutr 57: 192-203, 2015.

24. Eirheim HU, Bundgaard C and Nielsen HM: Evaluation of different toxicity assays applied to proliferating cells and to stratified epithelium in relation to permeability enhancement with glycocholate. Toxicol In Vitro 18: 649-657, 2004.

25. Spruss A, Kanuri G, Stahl C, Bischoff SC and Bergheim I: Metformin protects against the development of fructose-induced steatosis in mice: Role of the intestinal barrier function. Lab Invest 92: 1020-1032, 2012.

26. Haub S, Kanuri G, Volynets V, Brune T, Bischoff SC and Bergheim I: Serotonin reuptake transporter (SERT) plays a critical role in the onset of fructose-induced hepatic steatosis in mice. Am J Physiol Gastrointest Liver Physiol 298: G335-G344, 2010.

27. Sellmann C, Priebs J, Landmann M, Degen C, Engstler AJ, Jin CJ, Gärttner S, Spruss A, Huber O and Bergheim I: Diets rich in fructose, fat or fructose and fat alter intestinal barrier function and lead to the development of nonalcoholic fatty liver disease over time. J Nutr Biochem 26: 1183-1192, 2015.

28. Jourdan T, Godlewski G, Cinar R, Bertola A, Szanda G, Liu J, Tam J, Han T, Mukhopadhyay B, Skarulis MC, et al: Activation of the Nlrp3 inflammasome in infiltrating macrophages by endocannabinoids mediates beta cell loss in type 2 diabetes. Nat Med 19: 1132-1140, 2013.

29. Bergheim I, Weber S, Vos M, Krämer S, Volynets V, Kaserouni S, McClain CJ and Bischoff SC: Antibiotics protect against fructose-induced hepatic lipid accumulation in mice: Role of endotoxin. J Hepatol 48: 983-992, 2008.

30. Choi VM, Herrou J, Hecht AL, Teoh WP, Turner JR, Crosson S and Bubeck Wardenburg J: Activation of Bacteroides fragilis toxin by a novel bacterial protease contributes to anaerobic sepsis in mice. Nat Med 22: 563-567, 2016.

31. Wexler HM: Bacteroides: The good, the bad, and the nitty-gritty. Clin Microbiol Rev 20: 593-621, 2007.

32. Do MH, Lee E, Oh MJ, Kim Y and Park HY: High-glucose or -fructose diet cause changes of the gut microbiota and metabolic disorders in mice without body weight change. Nutrients 10: E761, 2018.

33. Mastrocola R, Ferrocino I, Liberto E, Chiazza F, Cento AS, Collotta D, Querio G, Nigro D, Bitonto V, Cutrin JC, et al: Fructose liquid and solid formulations differently affect gut integrity, microbiota composition and related liver toxicity: A comparative in vivo study. J Nutr Biochem 55: 185-199, 2018. 